\title{
Improvement of Anode/HTL Interface Properties Using Self-Assembled Monolayer in Organic Electronic Devices
}

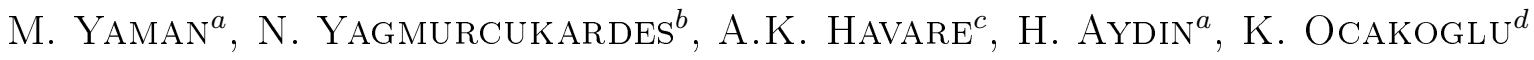 \\ AND S. OKUR ${ }^{e}$
}

${ }^{a}$ Ondokuz Mayıs University, Science and Arts Faculty, Department of Physics, Samsun, Turkey ${ }^{b}$ Izmir Institute of Technology, Faculty of Science, Material Science and Engineering, Izmir, Turkey ${ }^{c}$ Toros University, Faculty of Engineering, Mersin, Turkey

${ }^{d}$ Mersin University, Advanced Technologies Research and Application Center, Ciftlikkoy, Turkey ${ }^{e}$ Izmir Katip Celebi University, Material Science and Engineering, Çiğli, İzmir, Turkey

\begin{abstract}
Fabrication and characterization of highly efficient organic light-emitting diode with surface modification of indium tin oxide anodes by using self-assembled monolayer technique have been studied. Four different self-assembled molecules, K-28 ruthenium complex, octadecylamine hydrochloride, octadecyltrichlorosilane and mercaptohexdecanoic acid are used to modify ITO surface to improve the interface properties. Space charge limited currents measurements have been used to evaluate carrier mobility under steady state current. The results show that the surface properties such as the stability of ITO anode layer have significant effects on charge injection in organic light-emitting diode devices.
\end{abstract}

DOI: $10.12693 /$ APhysPolA.123.459

PACS: 73.40.Qv

\section{Introduction}

Indium tin oxide (ITO) coated glass substrates with high optical transparency and good electrical conductivity are widely used as anode layer in light emitting diode applications [1, 2]. The surface properties of the ITO anode layer have significant effects on charge (hole) injection and LED lifetime. Therefore, self-assembled monolayers (SAMs) are used to modify ITO surface to improve the interface properties [3-7] between ITO and hole transport layers (HTL) such as $N, N^{\prime}$-bis(3-methylphenyl)- $N, N^{\prime}$ -diphenylbenzidine (TPD) and $N, N^{\prime}$-di-[(1-naphthyl)$-N, N^{\prime}$-diphenyl]-1, 1'-biphenyl)-4, $4^{\prime}$-diamine (NPB) organic films.

At high voltages, $J-V$ characteristics switch to the space charge limited current (SCLC). At high voltages, the carriers eventually fill up all the trap states in the organic film and the organic LED (OLED) operates in a space-charge-limited conduction regime $[8,9]$. The field dependent SCLC can be expressed as follows:

$$
J=\frac{9}{8} \varepsilon \varepsilon_{0} \frac{E^{2}}{L} \mu_{0} \exp (\beta \sqrt{E}),
$$

where $E$ is the electric field, $\varepsilon$ and $\varepsilon_{0}$ are the relative dielectric constant and the permittivity of the free space, respectively, $L$ is the thickness of the organic layer, $\mu_{0}$ is the zero-field mobility and $\beta$ is the Poole-Frenkel factor. SCLC measurements have been used to evaluate carrier mobility under steady state current in an organic layer to evaluate the hole mobility of hole transport layer NPB which we used in this study with SAM molecules K-28 ruthenium complex, octadecylamine hydrochloride (ODA-HCl), octadecyltrichlorosilane (ODT-Cl-Si) and mercaptohexdecanoic acid.
On the other hand, the Schottky barrier can be mentioned at the metal/organic interface due to different energy level alignments. The current voltage characteristic for $q\left(V-I R_{\mathrm{s}}\right)>k T$ values of the diode is analyzed by the following relation [10]:

$$
I=I_{0} \exp \left(\frac{q\left(V-I R_{\mathrm{s}}\right)}{n k T}\right)\left[1-\exp \left(\frac{q\left(V-I R_{\mathrm{s}}\right)}{k T}\right)\right],
$$

where $I_{0}$ is the saturation current obtained from the intercept of the linear portion of $\log I$ at $V=0$ and expressed as $I_{0}=A A^{*} T^{2} \exp \left(-\frac{q \phi_{\mathrm{B}}}{k T}\right), n$ is the ideality factor, $k$ is the Boltzmann constant, $q$ is the electronic charge, $A$ is the contact area, $A^{*}$ is the Richardson constant, $T$ is the temperature and $\phi_{\mathrm{B}}$ is the barrier height.

In our study, K-28 ruthenium complex molecules dissolved in ethanol solvent, ODA-HCl molecules dissolved in methanol solvent, ODT-Cl-Si molecules dissolved in chloroform solvent, and mercaptohexdecanoic acid molecules dissolved in ethanol solvent are used as SAM molecules to modify ITO anode surface. The Schottky barrier characterization and SCLC techniques are used to investigate electrical characterization of organic LEDs.

\section{Experimental techniques}

ITO coated glass slides (from Sigma-Aldrich) with a surface resistivity of $15-25 \Omega / \mathrm{sq}$ were used as the substrates. NPB was used as HTL material (99\%, Aldrich Co.) and tris-(8-hydroxyquinoline) aluminum $\left(\mathrm{Alq}_{3}\right)$ was used as emissive layer (EML) material (99.995\% trace metals basis, Aldrich). Because of silanes, acids and amines can bind ITO surface chemically, in this 
study synthesized the ruthenium(II) bipyridyl complex K-28 molecule [11] and commercial molecules mercaptohexdecanoic acid, ODT-Cl-Si, and ODA-HCl (Sigma-Aldrich) were used as SAM molecules illustrated in Fig. 1, respectively.
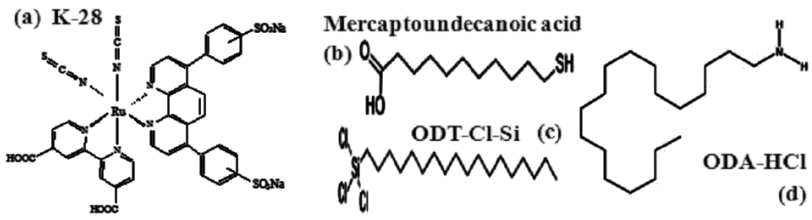

Fig. 1. Chemical structures of K-28 (a), mercaptoundecanoic acid (b), ODT-Cl-Si (c) and ODA$-\mathrm{HCl}(\mathrm{d})$.

$15 \mathrm{~mm} \times 15 \mathrm{~mm}$ pieces of ITO-coated glass samples were etched using zinc powder and $20 \% \mathrm{HCl}$ acid solution. In an attempt to clean substrates, each etched ITO slide was swabbed by rubbing with acetone, followed by a sequence of 30 min ultrasonic baths in detergent in de-ionized water, de-ionized water, acetone, ethanol, iso-propanol, and finally, de-ionized water again. In addition, oxygen plasma method is applied on the ITO surface for $15 \mathrm{~min}$ in order to clean the substrate surfaces from volatile species and organic molecules using $14 \% \mathrm{O}_{2}$ and $86 \%$ Ar gas mixture. ITO substrates were left into SAM solvent concentrations and were kept fixed as $1 \mathrm{mM}$ for all SAMs for $48 \mathrm{~h}$. Then, modified ITO substrates were rinsed with their pure solvent and finally they were dried with stream of $\mathrm{N}_{2}$ gas. After the surface modification, $50 \mathrm{~nm}$ thick NBP layer was deposited at a rate of $2-3 \AA \mathrm{s}^{-1}$ followed by $40 \mathrm{~nm}$ thick $\mathrm{Alq}_{3}$ that was used as EML deposited at a rate of $1-2 \AA \mathrm{s}^{-1}$. Finally, $200 \mathrm{~nm}$ thick Al (99\% trace metals basis) was deposited to form the top contact cathode layer at a rate of $5-10 \AA \mathrm{s}^{-1}$ in thermal evaporation coater from NANOVAK Co., Turkey.

\section{Results and discussions}

The Schottky characteristics of the devices were investigated to calculate barrier height after the surface modification with SAM molecules and HTL materials. Current-voltage characteristics of the diodes are shown in Fig. 2a.

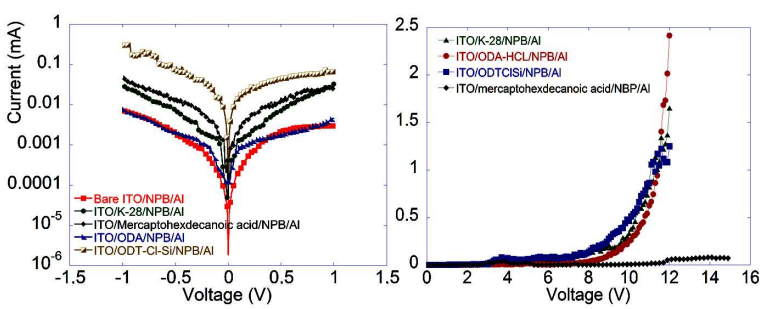

Fig. 2. Current-voltage characteristics of the fabricated diodes.

Carrier mobilities of the fabricated devices under steady state current are obtained using the currentvoltage characteristics. They are presented in Fig. 2 b.
Calculated charge carrier mobilities of the fabricated devices are presented in Table. According to these results it is obvious that carrier mobilities enhanced with SAM modification. The best improvement is achieved with mercaptohexdecanoic acid modification.

TABLE

CAlculated barrier height and hole mobility vAlues of the fabricated diodes.

\begin{tabular}{l|c|c}
\hline \hline \multicolumn{1}{c|}{ Diodes } & $\begin{array}{c}\text { Barrier } \\
\text { height, } \\
\phi_{b}[\mathrm{eV}]\end{array}$ & $\begin{array}{c}\text { Carrier } \\
\text { mobility } \\
{\left[\mathrm{cm}^{2} /(\mathrm{V} \mathrm{s})\right]}\end{array}$ \\
\hline bare ITO/NPB/Al & 0.491 & $4.6 \times 10^{-6}$ \\
ITO/K-28/NPB/Al & 0.448 & $2.3 \times 10^{-5}$ \\
ITO/ODA-HCl/NPB/Al & 0.469 & $2.8 \times 10^{-5}$ \\
ITO/ODTClSi/NPB/Al & 0.416 & $2.2 \times 10^{-5}$ \\
ITO/mercaptohexdecanoic acid/NPB/Al & 0.418 & $3.2 \times 10^{-5}$
\end{tabular}

\section{Conclusion}

Surface morphology, surface potential and currentvoltage characteristics of the devices with/without SAM modifications using $\mathrm{K}-28$, ODA-HCl, ODT-Cl-Si and mercaptohexdecanoic acid molecules are investigated. Our results indicate that SAM modification improved the surface properties of the films. Additionally, currentvoltage results show that SAM modification reduced the interface mismatch by lowering barrier height and by increasing the carrier transportation with higher charge carrier mobilities.

\section{Acknowledgments}

This work was supported by TUBITAK under grant No. TBAG-108T718.

\section{References}

[1] G. Gu, V. Khalfin, S.R. Forrest, Appl. Phys. Lett. 73, 2399 (1998)

[2] C. Yan, M. Zharnikof, A. Goölzhaäuser, M. Grunze, Langmuir 16, 6208 (2000).

[3] J.S. Kim, M. Granstroöm, R.H. Friend, N. Johansson, W.R. Salaneck, R. Daik, W.J. Feast, F. Cacialli, J. Appl. Phys. 84, 6859 (1998)

[4] Q. Huang, G.A. Evmenenko, P. Dutta, P. Lee, N.R. Armstrong, T.J. Marks, J. Am. Chem. Soc. 127, 10227 (2005)

[5] K.-Y. Wu, Y.-T. Tao, H.-W. Huang, Appl. Phys. Lett. 90, (2007)

[6] J. Lee, B.-J. Jung, J.I. Lee, H.Y. Chu, L.-M. Do, H.-K. Shim, J. Mater. Chem. 12, 3494 (2002).

[7] J.-P. Hong, A.-Y. Park, S.H. Lee, J.H. Kang, N. Shin, D.Y. Yoon, Appl. Phys. Lett. 92, 143311 (2008).

[8] T.Y. Chu, O.K. Song, Appl. Phys. Lett. 90, 203512 (2007)

[9] M.A. Khan, W. Xu, Y. Bai, X.Y. Jiang, Z.L. Zhang, W.Q. Zhu, J. Appl. Phys. 103, 014509 (2008)

[10] E.H. Rhoderick, R. Williams, Metal-Semiconductor Contacts, Clarendon Press, Oxford 1988.

[11] K. Ocakoğlu, C. Zafer, B. Çetinkaya, S. İçli, Dyes Pigments 75, 385 (2007) 